\title{
CytoTrack Analysis Reveals Low Presence of Circulating Tumor Cells in the Perioperative Period in Patients with Non-metastatic Colorectal Cancer
}

\author{
MIKAIL GOGENUR ${ }^{1}$, THORE HILLIG ${ }^{2}$ and ISMAIL GOGENUR ${ }^{1}$ \\ ${ }^{1}$ Center for Surgical Science Department of Surgery, Zealand University Hospital, Koge, Denmark; \\ ${ }^{2}$ Department of Clinical Biochemistry, Hillerod Hospital, Hillerod, Denmark
}

\begin{abstract}
Background/Aim: Detection of circulating tumor cells in the perioperative period predicts poor prognosis in patients with colorectal cancer. There is only one Food and Drug Administration-approved method for such detection, CellSearch, for which results have been inconsistent. A new immunological method, CytoTrack, has shown promising results in patients with breast cancer. The aim of this study was to evaluate detection of circulating tumor cells in the peripheral blood of patients with non-metastatic colorectal cancer with CytoTrack, and investigate if there is a correlation between presence of circulating tumor cells and Union for International Cancer Control (UICC) stage and if surgery itself results in the release of circulating tumor cells. Patients and Methods: A prospective study was conducted including patients with colorectal cancer UICC stage I-III who underwent minimally-invasive surgery. Patients with previous cancer diagnosis or neoadjuvant chemo/ radiotherapy were excluded. Blood samples were collected from all included patients one day prior to and one day after surgery. Detection of circulating tumor cells was performed using CytoTrack. Results: A total of 20 patients were consecutively included. Circulating tumor cells were detected in one preoperative sample and two postoperative samples. Conclusion: The presence of circulating tumor cells is rare in patients with non-metastatic colorectal cancer and the new method we used, CytoTrack, was only able to detect circulating tumor cells in three out of 40 blood samples. More specific antibodies are needed to identify circulating tumor cells in these patients.
\end{abstract}

Correspondence to: Mikail Gögenur, BMSc, Department of Gastrointestinal Surgery, Zealand University Hospital, Lykkebækvej 1, 4600 Køge, Denmark. E-mail: mgog@regionsjaelland.dk

Key Words: Colorectal cancer, non-metastatic circulating tumor cells, CytoTrack analysis, perioperative period.
The hypothesis of the existence of circulating tumor cells (CTCs) was proposed as early as 1869 (1) and further elucidated in 1955 (2). Studies have shown that healthy individuals or patients with non-malignant disease have no CTCs in their blood compared with patients with carcinoma (3). It has been established that surgery induces increased dissemination of CTCs (4). A meta-analysis found that detection of CTCs in the perioperative period predicts poor prognosis in patients with colorectal cancer (5). Different techniques have been used to detect such cells (6-8). The only Food and Drug Administration approved method, CellSearch, delivers inconsistent results with regards to peripheral blood samples $(9,10)$. A new method, CytoTrack, was recently verified in studies in patients with breast cancer $(11,12)$. This method is based on staining tumor cells with different specific antibodies and fluorescence analysis without prior enrichment of CTCs in the sample. The aim of this study was to explore if CytoTrack could detect CTCs in patients before or after surgery for non-metastatic colorectal cancer.

\section{Patients and Methods}

Study design and patients. Prior to inclusion of patients, the method was tested in 36 healthy donors, after obtaining their written informed consent.

This prospective study was conducted at a single center between March and June 2016. Patients with non-metastatic colorectal cancer who underwent curative surgery with the laparoscopic procedure were eligible. The inclusion criteria were: signed informed consent and colorectal cancer diagnosis either verified by computed tomographic scan, histological verification or clinical cancer diagnosis. Exclusion criteria were neoadjuvant chemo/radiotherapy, previous cancer diagnosis, or stage IV cancer.

The study was approved by the regional Ethics Committee (approval number \#49412). The patient characteristics (age, gender, tumor location, Union for International Cancer Control (UICC) stage, type of surgery, type of anesthesia, and length of stay) were collected. The tumors were classified according to the fifth edition of the TNM classification of the UICC (13). 
Table I. Patient and pathological characteristics.

\begin{tabular}{lc}
\hline Characteristic & Value \\
\hline Gender (male), n (\%) & $14(70.00 \%)$ \\
Median age (range), years & $66(54-85)$ \\
Median weight (range), kg & $73(55-111)$ \\
Median height (range), cm & $172(153-192)$ \\
T-Stage* & \\
n.a. & $5(25.00 \%)$ \\
$<$ T2 & $9(45.00 \%)$ \\
T3 & $5(25.00 \%)$ \\
T4 & $1(5.00 \%)$ \\
Tumor localization & \\
Right-sided & $5(25.00 \%)$ \\
Left-sided & $9(45.00 \%)$ \\
Rectum & $6(30.00 \%)$ \\
UICC stage & \\
I & $5(25.00 \%)$ \\
IIA & $8(40.00 \%)$ \\
IIIA & $1(5.00 \%)$ \\
IIIB & $5(25.00 \%)$ \\
IIIC & $1(5.00 \%)$ \\
\hline
\end{tabular}

*By computed tomography. n.a. Not applicable.
Table II. Surgical characteristics.

\begin{tabular}{lc}
\hline Characteristic & $\mathrm{n}(\%)$ \\
\hline Type of surgery & \\
Laparoscopic & $20(100.00 \%)$ \\
Conversion & 0 \\
Robot-assisted & $4(20.00 \%)$ \\
Stoma & $16(80.00 \%)$ \\
$\quad$ No stoma & $4(20.00 \%)$ \\
Temporary stoma & \\
Length of stay & $13(65.00 \%)$ \\
$\leq 4$ Days & $7(35.00 \%)$ \\
$>4$ Days & \\
Anesthesia & $13(65.00 \%)$ \\
Total intravenous & $7(35.00 \%)$ \\
Gas & \\
Anastomotic leak & $4(20.00 \%)$ \\
Yes & $16(80.00 \%)$ \\
No & $14(70.00 \%)$ \\
Blood loss & $4(20.00 \%)$ \\
$<10 \mathrm{ml}$ & $2(10.00 \%)$ \\
$10-100 \mathrm{ml}$ & 0 \\
$100-500 \mathrm{ml}$ & \\
$>500 \mathrm{ml}$ &
\end{tabular}

Isolation and enumeration of CTCs. Blood samples were collected one day prior to and one day after surgical procedure. Blood was collected in $10 \mathrm{ml}$ tubes (CellSave, Immunicon, Huntingdon Valley, PA, USA). The samples were stored at room temperature, mailed within 2 days and processed within 1 week. All CTC evaluations were blinded from laboratory to patient clinical status.

The CytoTrack method has been published in length elsewhere (11). In short, blood samples were centrifuged for $15 \mathrm{~min}$ at 2500 $\times \mathrm{g}$ and the buffy coat layer of cells isolated. Red blood cells in the isolated cells were lysed with FACS lysing solution (BD Biosciences, San Jose, CA, USA) for 15 min during gentle mixing. After a centrifugation step $(15 \mathrm{~min}$ at $2,500 \times g)$ cells were stained with a modified CTC Stain ${ }^{\mathrm{TM}}$ comprising a mixture of antiCD45/APC antibody (clone: HI30), anti-pancytokeratin/ AlexaFluor488 antibody (clone: AE1/AE3), anti-carcinoembryonic antigen (CEA/AlexaFluor488 antibody (clone: CEA31), anti-caudal type homeobox 2 (CDX2)/AlexaFluor488 antibody (clone: DAKCDX2), 4',6-diamidino-2-phenylindole (DAPI), and a permeabilization buffer (Intrastain; Agilent Technologies, Santa Clara, CA, USA). Cells were then washed with phosphate-buffered saline with $1 \%$ and resuspended in water, transferred to a CytoDisc $^{\mathrm{TM}}$, air-dried, and mounted using VectaShield Hard Set Mounting Medium (Vector Laboratories Inc., Burlingame, CA, USA) and covered with a CytoCover ${ }^{\mathrm{TM}}$. The CytoDiscs ${ }^{\mathrm{TM}}$ were scanned for CTCs in a CytoTrack ${ }^{\mathrm{TM}}$ scanning fluorescence microscope in a $10-\mu \mathrm{m}$ band width spiral scanning pattern with excitation by a $488-\mu \mathrm{m}$ laser $(20 \mathrm{~mW})$ using scanning parameters for AlexaFluor488 signals: threshold $0.08 \mathrm{mV}$, width $70 \mu \mathrm{m}$, symmetry $50 \%$. Fluorescence signals conforming to the scanning parameters were listed in a hotspot table and manually inspected under microscopy. Definition of a CTC was a cell: $>4 \mu \mathrm{m}$ diameter, DAPI-positive, CK-positive, CD45-negative.
Statistical analysis. Median, range, and descriptive statistics calculations were performed using SAS version 9.4 windows (SAS Institute, Cary, MC, USA).

\section{Results}

In 36 healthy donors, no CTCs were found except in two donors for whom two and seven cells were found to stain both cytokeratin and CD45. Twenty patients were included in the study, 14 males and six females, with a median age of 66 years (range $=54-85$ years). The patient characteristics are summarized in Table I. Data on surgery are presented in Table II. All patients underwent laparoscopic surgery with four patients undergoing robot-assisted laparoscopic procedure. Six patients had blood loss over $10 \mathrm{ml}$, while four patients had anastomotic leakage. Intraoperative carcinosis or tumor perforation was not discovered in any patient.

A total of 40 blood samples were collected. Non-specific cells positive for CD45 and epithelial cell antigen cytokeratin were detected in seven patients (Figure 1). CTCs were detected in one patient preoperatively but not postoperatively. This patient also had non-specific cells positive for CD45 and cytokeratin. One more patient had one CTC preoperatively and one CTC postoperatively. Due to the very low CTC yield, there was no statistical power to determine correlation between the number of CTCs in blood and UICC stage. 

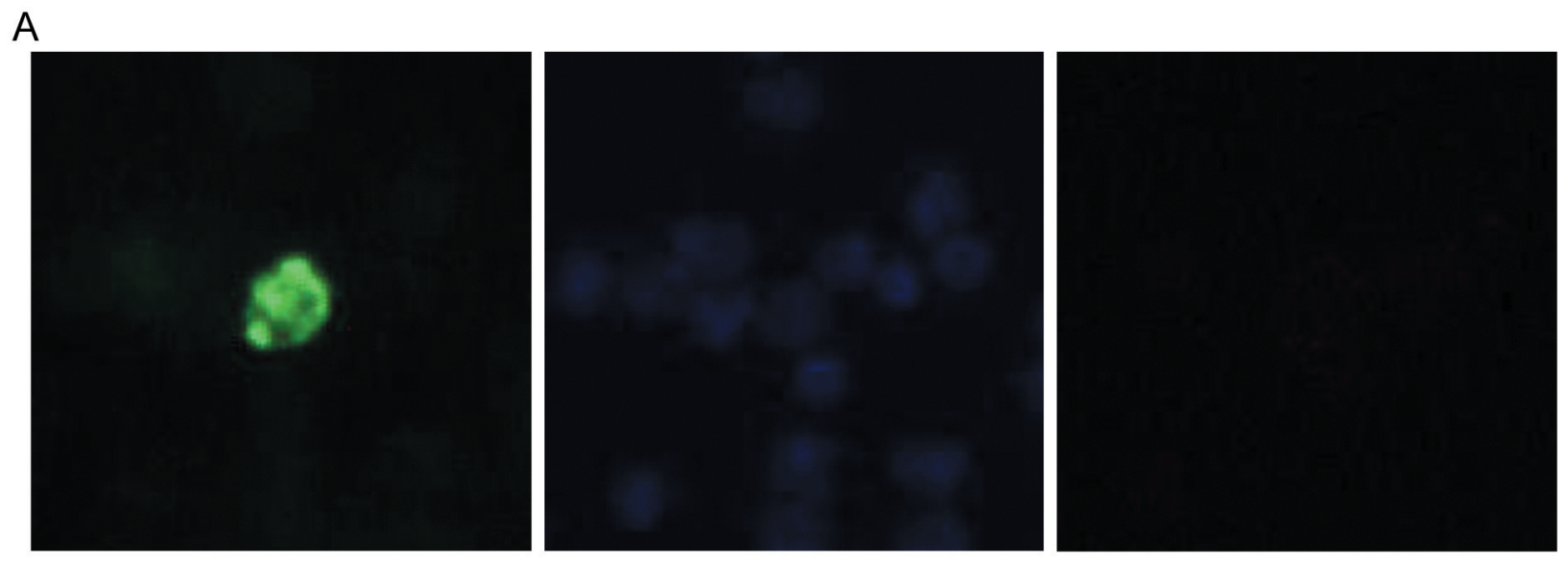

B
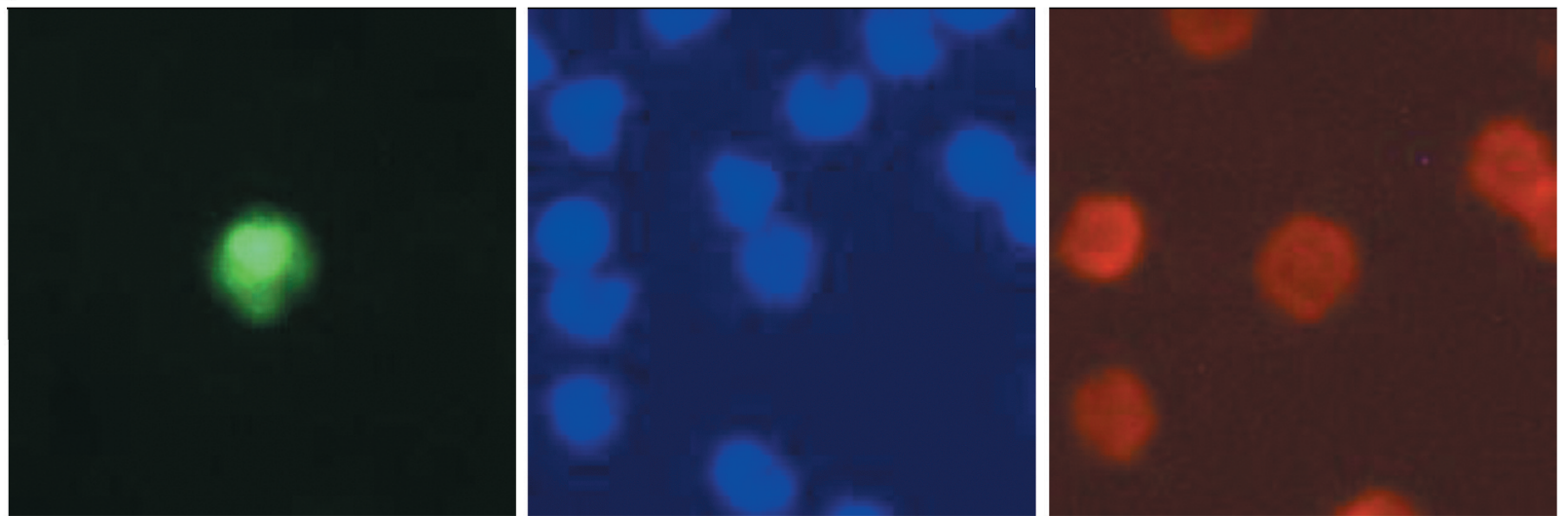

\section{C}
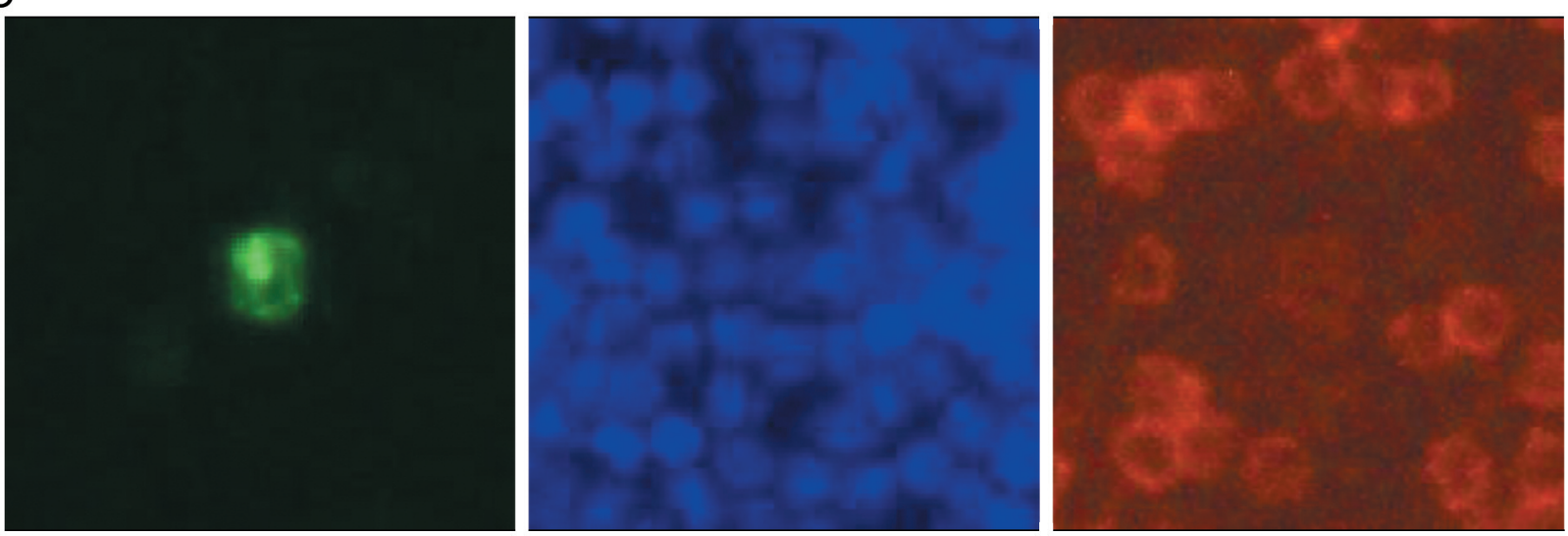

Figure 1. The columns depict the same cells stained with different markers. Left column: Staining with primary antibodies to pancytokeratin, carcinoembryonic antigen, and caudal type homeobox 2, all with AlexaFluor488 conjugation. Middle column: Staining with diamidino-2-phenylindole. Right column: Staining with anti-CD45 with allophycocyanin conjugation. A: A circulating tumor cell from a patient positive for cytokeratin and negative for CD45. B: A cell from a donor positive for cytokeratin and for CD45. C: A cell from a patient positive for cytokeratin and CD45.

\section{Discussion}

We conducted a prospective cohort study where we measured CTCs 1 day prior to and 1 day after surgery. We were able to detect CTCs in three preoperative and postoperative samples. Previous studies have shown that a higher number of CTCs in patients with colorectal cancer is correlated to the UICC stage of the cancer $(14,15)$. We found that our cohort consisted of 
$25 \%$ with UICC stage I, $40 \%$ with UICC stage IIA, and $35 \%$ with UICC stage IIIA-C, which are similar to other studies in this field. In a study where the reverse transcriptase method was used, preoperative CTCs were measured in 438 patients with UICC stage I-III cancer (6). Their results confirmed that CTCs are more frequently present in patients with UICC stage III compared to UICC stage I. Of their 438 patients, 184 patients were diagnosed with UICC stage III cancer. In this subgroup, they detected CTCs in $96.2 \%$ of preoperative samples, while only $41.3 \%$ were positive postoperatively. One important difference between their study and ours is that the postoperative sample was taken 1 week after surgery. Another study using a different method found similar results (16). It is however interesting to note that some studies in this field also had difficulty in detecting CTCs in patients with UICC stage III cancer. One study, with a similar prospective cohort design, using the CellSearch method found CTCs in only one patient (10).

CellSearch and CytoTrack both analyze CTCs by an immunological approach. They have been compared in a study using cells from patients with breast cancer, where they found similar results for the two methods (12). This leads to the important question: Is the immunological approach feasible in patients with colorectal cancer? To answer this question, it is important to consider which antibodies the two methods use and if these antibodies are sufficiently specific in the study settings. It is a well known phenomenon that cancer cells undergo epithelial to mesenchymal transition (EMT) (17). Studies have shown that cancer cells lose many of their epithelial characteristics such as cell adhesion, the repression of E-cadherin, and gain increased cell motility (17). It is hypothesized that this transition begins at the invasive front (18). Surgery then induces a dissemination of mesenchymal cancer cells to blood circulation as the physical manipulation of tumor tissue under surgery leads to increased levels of circulating cancer cells in blood (4). During and after this transition, the antigens expressed on the cancer cell surface undergo a transformation where, among others, the expression of cytokeratin is reduced (19). Both methods use the antibody anti-pancytokeratin in the staining of CTCs and may be a reason for the difficulty in detecting CTCs in patients with non-metastatic colorectal cancer. The degree of EMT is difficult to assess, since the cells lose expression of known markers which discerns them from leukocytes.

Another reason could be the low level of CTCs in CRC caused by the anatomy of colorectal blood supply, where venous blood passes through the portal vein and liver before entering the systemic circulation. Indeed, a study investigating CTCs in portal vein blood from CRC found markedly higher numbers of CTCs (20) in the portal circulation. In line with this, another study found that only central CTCs are prognostic in CRC (21). This is possibly because of the high levels of CTCs leading to a better certainty of detection of CTC-positive patients. Moreover, recent reports suggest that liver metastasis from CRC are the main contributors to CTC in systemic circulation $(15,22)$.

A curious finding in this study was the presence of cells positive for both CD45 and cytokeratin in seven patients. We do not know the nature of these cells but speculate they could either be leukocytes with phagocytosed CTC fragments, or CTCs in the process of EMT. However, these cells were also found at a low frequency in healthy donors, perhaps indicating these cells as benign. Are the prognoses for patients with these kind of cells affected? Further followup is needed to address this question.

This study had several strengths and limitations. The strength of the study was that we were able to take blood samples 1 day prior to 1 day after curative surgery and analyze the blood samples blinded to the patient clinical status. This study was limited by the number of patients who were eligible but were not included in the study for different reasons. A limitation in the analysis of CTCs by CytoTrack as well as other current CTC technologies is the use of a small blood volume $(7.5 \mathrm{ml})$ to detect very rare cells.

\section{Conclusion}

This study shows that although the CytoTrack method was verified in patients with breast cancer, we were only able to detect CTCs in 3 out of 40 blood samples from patients with non-metastatic colorectal cancer. We had, however, only 20 patients in the study, which warrants larger studies to conclude whether the CytoTrack method is feasible in patients with colorectal cancer.

\section{References}

1 Ashworth T: A case of cancer in which cells similar to those in the tumours were seen in blood after death. Aus Med J 14: 146149, 1869.

2 Engell H: Cancer cells in circulating blood; a clinical study on the occurrence of cancer cells in the peripheral blood and in venous blood draining the tumour are at operation. Acta Chir Scand Suppl 201: 1-70, 1955.

3 Allard W, Matera J, Miller M, Repollet M, Connelly M, Rao C, Tibbe A, Uhr J and Terstappen L: Tumor cells circulate in the peripheral blood of all major carcinomas but not in healthy subjects or patients with nonmalignant diseases. Clin Cancer Res 10: 6897-6904, 2004.

4 Hayashi N, Egami H, Kai M, Kurusu Y, Takano S and Ogawa $\mathrm{M}$ : No-touch isolation technique reduces intraoperative shedding of tumor cells into the portal vein during resection of colorectal cancer. Surgery 125: 369-374, 1999.

5 Rahbari N, Aigner M, Thorlund K, Mollberg N, Motschall E, Jensen K, Diener M, Büchler M, Koch M and Weitz J: Metaanalysis shows that detection of circulating tumor cells indicates poor prognosis in patients with colorectal cancer. Gastroenterology 138: 1714-1726, 2010. 
6 Uen Y, Lu C, Tsai H, Yu F, Huang M, Cheng T, Lin S and Wang $\mathrm{J}$ : Persistent presence of postoperative circulating tumor cells is a poor prognostic factor for patients with stage I-III colorectal cancer after curative resection. Ann Surg Oncol 15: 2120-2128, 2008.

7 Cristofanilli M, Budd G, Ellis M, Stopeck A, Matera J, Miller M, Reuben J, Doyle G, Allard W, Terstappen L and Hayes D: Circulating tumor cells, disease progression and survival in metastatic breast cancer. N Engl J Med 351: 781-791, 2004.

8 Weitz J, Kienle P, Lacroix J, Willeke F, Benner A, Lehnert T, Herfarth C and Doeberitz M: Dissemination of tumor cells in patients undergoing surgery for colorectal cancer. Clin Cancer Res 4: 343-348, 1998.

9 Wind J, Tuynman J, Tibbe A, Swennenhuis J, Richel D, Henegouwen $\mathrm{M}$ and Bemelman W: Circulating tumour cells during laparoscopic and open surgery for primary colonic cancer in portal and peripheral blood. Eur J Surg Oncol 35: 942-950, 2009.

10 Thorsteinsson M, Söletormos G and Jess P: Low number of detectable circulating tumor cells in non-metastatic colon cancer. Anticancer Res 31: 613-617, 2011.

11 Hillig T, Nygaard A, Nekiunaite L, Klingelhöfer $\mathrm{J}$ and Söletormos G: In vitro validation of an ultra-sensitive scanning fluorescence microscope for analysis of Circulating Tumor Cells. APMIS 122: 545-551, 2014.

12 Hillig T, Horn P, Nygaard A, Haugaard A, Nejlund S, Brandslund I and Söletormos G: In vitro detection of circulating tumor cells compared by the CytoTrack and CellSearch methods. Tumor Biol 36: 4597-4601, 2015.

13 Sobin LH and Fleming ID: TNM Classification of Malignant Tumors, fifth edition (1997). Union Internationale Contre le Cancer and the American Joint Committee on Cancer. Cancer 80: 1803-1804, 1997.

14 Bork U, Rahbari N, Schölch S, Reissfelder C, Kahlert C, Büchler M, Weitz J and Koch M: Circulating tumour cells and outcome in non-metastatic colorectal cancer: a prospective study. Br J Cancer 112: 1306-1313, 2015.

15 Rahbari N, Bork U, Schölch S, Reissfelder C, Thorlund K, Betzler A, Kahlert C, Schneider M, Ulrich A, Büchler M, Weitz $\mathrm{J}$ and Koch M: Metastatic spread emerging from liver metastases of colorectal cancer. Ann Surg 263: 345-352, 2016.
16 Tsai W, Chen J, Shao H, Wu J, Lai J, Lu S, Hung T, Chiu Y, You J, Hsieh P, Yeh C, Hung H, Chiang S, Lin G, Tang R and Chang Y: Circulating tumor cell count correlates with colorectal neoplasm progression and is a prognostic marker for distant metastasis in non-metastatic patients. Sci Rep 6: 24517, 2016.

17 Sipos F and Galamb O: Epithelial-to-mesenchymal and mesenchymal-to-epithelial transitions in the colon. World $\mathrm{J}$ Gastroenterology 18: 601-608, 2012.

18 Hugo H, Ackland M, Blick T, Lawrence M, Clements J, Williams E and Thompson E: Epithelial-mesenchymal and mesenchymal-epithelial transitions in carcinoma progression. $\mathrm{J}$ Cell Physiol 213: 374-383, 2007.

19 Hay E: An overview of epithelio-mesenchymal transformation. Acta Anat 154: 8-20, 1995.

20 Jiao L, Apostolopoulos C, Jacob J, Szydlo R, Johnson N, Tsim $\mathrm{N}$, Habib N, Coombes C and Stebbing J: Unique localization of circulating tumor cells in patients with hepatic metastases. J Clin Oncol 27: 6160-6165, 2009.

21 Connor AA, McNamara K, Al-Sukhni E, Diskin J, Chan D, Ash C, Lowes LE, Allan AL, Zogopoulos G, Moulton C-A and Gallinger S: Central, but not peripheral, circulating tumor cells are prognostic in patients undergoing resection of colorectal cancer liver metastases. Ann Surg Oncol 23: 2168-2175, 2015.

22 Kaifi J, Kunkel M, Dicker D, Allen J, Das A, Zhu J, Yang Z, Sarwani N, Li G, Staveley-O'Carrol K and El-Deiry W: Circulating tumor cell levels are elevated in colorectal cancer patients with high tumor burden in the liver. Cancer Biol Ther 16: 690-698, 2015.
Received April 11, 2017

Revised April 24, 2017

Accepted April 28, 2017 